\title{
Ethics in the age of COVID-19
}

\author{
Ananya Arora ${ }^{1}$ (D) Anmol Arora $^{2}$ (1)
}

Received: 24 April 2020 / Accepted: 6 May 2020 / Published online: 21 May 2020

(c) Società Italiana di Medicina Interna (SIMI) 2020

\section{Dear Editor,}

The current COVID-19 pandemic is continuing to inflict unprecedented strain on healthcare systems worldwide. Hospitals are demonstrating great flexibility, with many outpatient consultations and elective operations widely cancelled. These decisions are not made lightly but as similar decisions are continued to be made, it is important to reflect on key ethical principles from the outset. The framework for ethical medical practice is governed by four key doctrines: autonomy, beneficence, non-maleficence and justice. These are essential components of clinical practice, especially when dealing with individual patients, but they may not provide enough guidance in unprecedented public health crises, such as the one we are currently dealing with.

Decisions around prioritising treatment are not new in healthcare, but the COVID-19 pandemic has highlighted these discussions in mainstream media and public debate. Scarcity of resources brings decisions involving conflicts of beneficence and non-maleficence between patients under great scrutiny. The pillar of justice is also under strain with a substantial amount of non-urgent care cancelled, which will surely have long-term implications. Amongst COVID19 patients, those with better expected outcomes following treatment will generally be prioritised for treatment escalation. There is a strong correlation between age and mortality and hence, age is an important, but not the sole, factor in prioritisation decisions $[1,2]$. This raises the question, would other protected characteristics such as sex, ethnicity or disability be acceptable to use in these decisions if

Anmol Arora

aa957@cam.ac.uk

Ananya Arora

aa2081@cam.ac.uk

1 University of Cambridge, Selwyn College, Grange Road, Cambridge CB3 9DQ, UK

2 School of Clinical Medicine, University of Cambridge, Addenbrooke's Hospital, Hills Road, Cambridge CB2 0SP, UK correlations with mortality were discovered? Furthermore, if care providers determine that a patient's outcomes appear less favourable than other candidates, should the patient have the autonomous right to demand ventilation? They may also face the ethical dilemma of whether they should withdraw support from a stable or even improving patient in order to provide it to another patient with more favourable outcomes [2]. These decisions are made even harder by the potentially rapid deterioration in COVID-19 and the risk that families may have less involvement in care planning due to visiting restrictions.

Priority decisions, if they are to be made, will be entrusted to experienced clinicians in trained teams who will use all available resources at their disposal. Yet the ambiguity and the potential scale for disruption has introduced unprecedented public scrutiny to the issue. There are also ethical questions revolving around persistent PPE issues, especially on the topic of how clinicians should act in situations where they do not have adequate PPE immediately available. Should doctors withhold treatment if they do not have adequate protective equipment? What are the legal ramifications of doing so, or not? To deal with such ethicolegal issues, doctors should be provided with clear guidance to govern, unify and defend their decisions. It is especially important that guidance continues to evolve as the context of clinical practice changes and we learn more about the emergent disease. As new research emerges regarding PPE effectiveness and supply, changes to practice may be warranted. Guidelines should also reflect that there should be scope for doctors to exercise their own professional judgement, without fear of unreasonable retribution. Traditionally, doctors have relied on the conventional aforementioned 'four pillars' for these principles. Unfortunately, it may be argued that in the face of a global pandemic the conventional 'four pillars' framework may be insufficient.

Author contributions Both Anmol Arora and Ananya Arora contributed to the conception of the article and both contributed equally to the 
writing and preparation. Both authors also reviewed and approved the manuscript prior to sending for publication.

Funding Not applicable.

Data availability Not applicable.

\section{Compliance with ethical standards}

Conflict of interest The authors report no conflict of interest.

Ethics approval Not applicable.

Consent to participate Not applicable.

Consent for publication Not applicable.

\section{References}

1. Ruan S (2020) Likelihood of survival of coronavirus disease 2019. The Lancet Infect Dis. https://doi.org/10.1016/S1473 -3099(20)30257-7

2. British Medical Association. COVID-19-ethical issues. A guidance note. Available from: https://www.bma.org.uk/media/2226/ bma-covid-19-ethics-guidance.pdf. Accessed 8th Apr 2020.

Publisher's Note Springer Nature remains neutral with regard to jurisdictional claims in published maps and institutional affiliations. 